\title{
BRONCHIAL ARTERY EMBOLIZATION AS A THERAPEUTIC STRATEGY FOR MANAGEMENT OF MASSIVE HEMOPTYSIS
}

\author{
Laila Ahmad Abdurrahman*; Ayman Mohamed Ibrahim*; \\ Shaimaa Abdelsattar Mohammad*and Heba Mounir Abdel Aziz**
}

\author{
*Department of Radiodiagnosis; \\ Faculty of Medicine - Ain Shams \\ University ** Radiodiagnosis \\ Specialist, Ministry of Health, \\ Cairo, Egypt. \\ Corresponding author \\ Heba Mounir Abdel Aziz \\ Mobile: (+2) 01556288506 \\ E.mail: \\ monier_heba@yahoo.com \\ Received: 18/5/2021 \\ Accepted: 24/6/2021
}

\section{Online ISSN: 2735-3540}

\begin{abstract}
:
Background: Massive hemoptysis is an emergent and lifethreatening condition with a high mortality rate. Bronchoscopy and MDCT scanning show significant contributing roles in delineating the etiology and the source of hemoptysis prior to bronchial artery arteriography.
\end{abstract}

Aim of the work: To validate the role of the trans-catheter bronchial artery embolization in management of massive hemoptysis.

Patients and Methods: Our study is a prospective cohort study that was held during the period between April 2017 and April 2019. The study included 20 patients who presented with massive hemoptysis refractory to supportive treatment measures with nonidentifiable source of bleeding on fiber-optic bronchoscope. Patients were referred from chest department to angio unit.

Results: Our findings are in accordance with the current literature supporting bronchial artery embolization as a safe and long-term non-invasive effective method of treatment for massive hemoptysis with a high initial rate of success. BAE may help to avoid surgery in patients who are not good surgical candidates. If hemoptysis recur in these patients, embolization can be safely performed with good response rate. If surgery is indicated, BAE can stabilize the patient prior to surgery.

Conclusion: Advancements in angiographic equipment and technique continue to improve success rates, and with careful technique, it can be performed safely and with minimal risk. Embolization distal to the spinal artery may significantly decrease the number of complications and may allow a more thorough embolization.

Keywords: Bronchial artery embolization; massive hemoptysis

\section{INTRODUCTION:}

Life threatening massive hemoptysis is one of the most challenging situations that is encountered in critical are necessitating an appropriate investigation. Regardless of advances in medical and emergency until management, massive hemoptysis endures a grave threat ${ }^{(1)}$.

Hemoptysis, when massive and left untreated, has a mortality rate of $>50 \%$ Thus; it demands prompt identification of the hemorrhage source so that definitive therapy can be commenced to cease the bleeding $^{(2)}$.

Death is predominantly due to asphyxiation resultant from blood aspiration, ending in airway obstruction ${ }^{(3)}$. As per literature, $28 \%$ of the pulmonologists had experienced a patient's death from significant hemoptysis during a year period ${ }^{(1)}$.

Ninety percent of life threatening hemoptysis is of bronchial artery origin ${ }^{(4)}$. 
However, non-bronchial systemic arteries can be also a substantial source and a reason for recurrence after effective bronchial artery embolization (BAE) ${ }^{(5)}$.

The presently available approaches to patients with hemoptysis are conservative treatment, BAE, and surgery. Chossing the appropriate treatment relies primarily on the severity and urgency of the condition ${ }^{(6)}$.

Conservative management of significant hemoptysis inclusive of observation and medication carries a mortality rate of $50 \%$ $100 \%{ }^{(1)}$.

This is understandable that the patient population susceptible to hemoptysis often additionally have previous medical condition further compromising the patient's situation $^{(7)}$.

Historically, surgical management had been the definitive therapy. Unluckily, surgical intervention carries a mortality of $\sim$ $18 \%$ when done electively, reaching up to $40 \%$ when performed on emergent basis ${ }^{(7)}$.

Owing to poor pulmonary reserve adding to other comorbid medical conditions, most patient with massive hemoptysis are not surgical candidates ${ }^{(1)}$.

BAE is regarded as the therapeutic method of choice for management of massive hemoptysis ${ }^{(8)}$. As a minimally invasive procedure, it can be presented to even the most debilitated patient serving as first-line treatment for hemorrhage as well as providing more definitive medical or nonemergent surgical intervention centered upon aetiopathogenesis of the hemorrhage ${ }^{(7)}$.

\section{AIM OF THE WORK:}

The aim of this study is to validate the role of the trans-catheter bronchial artery embolization in management of massive hemoptysis.

\section{PATIENTS AND METHODS:}

Our study is a prospective cohort study that was held during the period between April 2017 and April 2019. The study included 20 patients who presented with massive hemoptysis refractory to supportive treatment measures with non-identifiable source of bleeding on fiber-optic bronchoscope. Patients were referred from chest department to angio unit.

Patients were enrolled in the study according to the following criteria: Age ranged from 43-79 years with no sex predilection. Patients were included upon having the following items: Massive hemoptysis; defined as coughing of about $300-600 \mathrm{ml}$ of blood/24hours or $200 \mathrm{ml}$ of blood/ hour that might be preceded by recurrent mild or moderate attacks of hemoptysis with concomitant (Hypo-tension where systolic blood pressure measures less than $100 \mathrm{mmHg}$ and Rapid drop in hemoglobin level of $1.0-2.0 \mathrm{mg} / \mathrm{dl} / \mathrm{day}$ ), and Failure of supportive or endobronchial measures to control the bleeding.

Any patient harboring one or more of the following criteria was excluded: Nonpulmonary cause of hemoptysis e.g. cardiovascular cause, traumatic hemoptysis or systemic cause attributable to coagulopathies and blood disorders, uncooperative patients, pregnant patients, severe renal impairment with high serum Creatinine reaching up $>2 \mathrm{mg} / \mathrm{dl}$, patients previously had life threatening contrast allergy, feeding vessels feed important structures as spinal cord with no option to avoid probable ischemia, vascular anatomy that is difficult for endovascular access; like patient having exaggerated vascular tortuosity, bacteremia at time of endovascular treatment, and significant atherosclerotic disease, or high flow vasculopathy affecting the parent vessel. 


\section{Technique of bronchial artery embolization:}

\section{Patient preparation:}

Proper history taking and full clinical assessment, pre-procedural assessment of imaging data including chest radiography, CT chest coupled with multidetector CT angiography to map bronchial arterial vasculature as well as results of fiber-optic bronchoscopy, investigating renal function inclusive of blood urea \& serum Creatinine, laboratory investigation encompassing cbc, ESR, ABG, coagulation profile, liver functions testing, virology markers, and laboratory assessment of activity of tuberculosis, administration of an antecubital intravenous catheter, and detailed explanation of imaging procedure with obtaining written informed consent comprising indication, outcome and possible complications.

\section{Treatment strategy:}

Based on CT angiography interpretation prior to the embolization procedure; decisions about the overall treatment strategy and the role of embolization should be made well ahead of time. Plans should include: Choice of access vessel, guide catheter selection, micro catheter and micro-wire selection, embolic agent to be used, and target vessels to be treated.

\section{Method:}

Vascular access phase: This phase involves accessing the arterial system via placement of a $5 \mathrm{~F}$ arterial introducer (Radifocus, TERUMO) at the right femoral artery under local anesthesia/ conscious sedation or general anesthesia in selected cases according to the patients' clinical condition.

Bronchial angiography: Aided by a $5 \mathrm{~F}$ Cobra2 Catheter or 5FR SIM2 catheter (Cordis) that is navigated over a $0.35 \mathrm{~F}$ hydrophilic guide wire (Radifocus; TERUMO), selective cannulation of bronchial arteries is done. A gentle injection of full syringe contrast under fluoroscopy is done, to examine the configuration of the vessel around the tip, to check for the presence of vasospasm or vessel dissection around the tip as well as determine type of angiographic pattern that predispose to hemoptysis.

Micro catheter access phase: Once a stable guide catheter position is achieved, a micro catheter (Renegade) is coaxially advanced to a position from which embolic material can be delivered to the target lesion.

Embolization phase: A variety of embolic agents such as Embosphere (500- 700 $\mu \mathrm{m})$, PVA-particles and Gel foam as well as pushable coils were delivered through micro catheters aiming at disappearance of vascular blush. The whole procedure could last from $30-60$ minutes.

Post- procedure outcome was evaluated in multiple aspects; technical, and clinical improvement. As a tool to assess technical success of BAE; control angiographies were assessed for final obliteration rate and lesion remnants if any. On clinical basis, follow-up of the patients' symptoms were monitored one month post-procedurally to check grade of hemoptysis resolution, necessity for reembolization session as well as stability of bleeding cessation. According to Society of Interventional Radiology (SIR) standards; complications were subdivided into:

(1) Major complication that necessitates longer hospital stay than expected or that leaves long-lasting adverse effect as spinal cord ischemia.

(2) Minor complications that can be self - limiting or solved on a day case admission.

\section{Statistical analysis:}

IBM SPSS statistics (V. 22.0, IBM Corp., USA, 2013) was used for data analysis. Data were expressed as Median and Percentiles for quantitative nonparametric measures and both number and percentage for categorized data.

\section{RESULTS:}

A total of 23 cases were gained from target population. Data from three patients were deficient as they missed their follow- 


\section{Laila Ahmad Abdurrahman et al.,}

up appointments; were discarded. Results from 20 patients were analyzed.

The male to female ratio in the study sample is $70 \%$ to $30 \%$ respectively. While the patient's age ranged between $40-79$ years with median age of 54 years (Range: 40-79 years) among males and 52.5 years among females (Range: 43- 64 years) (Table 1).

Table (1): Age and sex distribution in our study

\begin{tabular}{|c|c|c|c|c|}
\hline & Sex & N & Median age (Mean) $^{\text {Yrs }}$ & Range \\
\hline \multirow{2}{*}{ Age } & Male & 14 & $54(53.85)$ & $40-79$ \\
\cline { 2 - 5 } & Female & 6 & $52.5(53.2)$ & $43-64$ \\
\hline
\end{tabular}

Patients presented with massive hemoptysis defined as voluminous coughing of blood ranging between 300-700 ml with mean volume of $481 \mathrm{ml}$. Hemoptysis was associated with dyspnea in $60 \%$ of patients, systolic hypotension in $10 \%$ of cases whilst corresponding hemoglobin level decrease rapidly within 24 hours by 1.0-2.0 which was varied in our sample between 6.3-10.3 $\mathrm{mg} / \mathrm{dl}$ with mean value of $9.16 \mathrm{mg} / \mathrm{dl}$ necessitating blood transfusion in $15 \%$ of patients (Diagram 1).

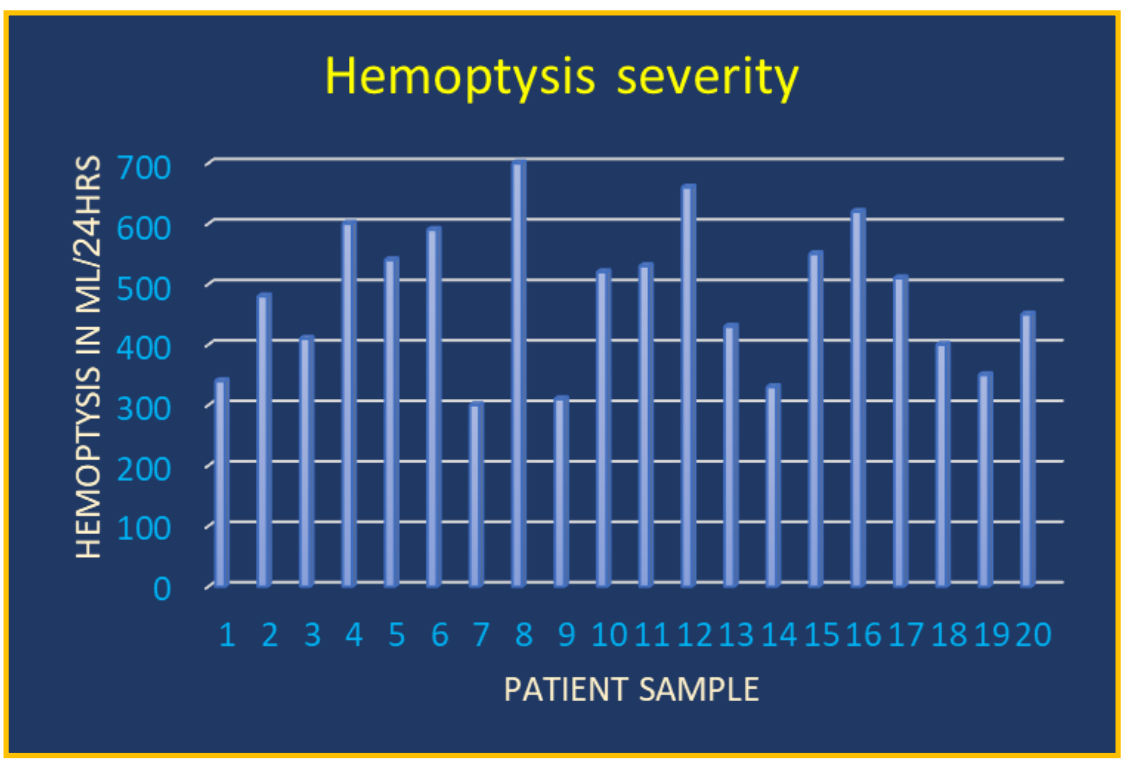

Diagram (1): Hemoptysis severity in study population.

Chest radiography was performed in $30 \%$ of our patients whilst all patients underwent chest CT coupled with CT Angiography to determine underlying pathology and to delineate source of bleeding. Cavitary lung lesion was the most prominent findings attributing to $25 \%$ of our cases. It ranged between aspergillosis (10\%), lung abscess $(10 \%)$, Post-TB sequel $(5 \%)$ followed by malignant etiology being represented by $20 \%$ divided between primary lung cancer in $15 \%$ and metastasis in 5\%. Pulmonary vascular etiologies and bronchiectasis occurred equally in $15 \%$ of patients. One patient exhibited ground-glass opacity in Chest CT corresponding to pulmonary alveolar hemorrhage. On the other hand, one patient presented with pulmonary fibrosis aggravated on right side sequent to interstitial lung disease (ILD). No underlying source of hemoptysis was denoted in $10 \%$ of cases whilst iatrogenic hemoptysis took place in one patient following lung biopsy performed for a suspicious pulmonary nodule (Diagram 2). 


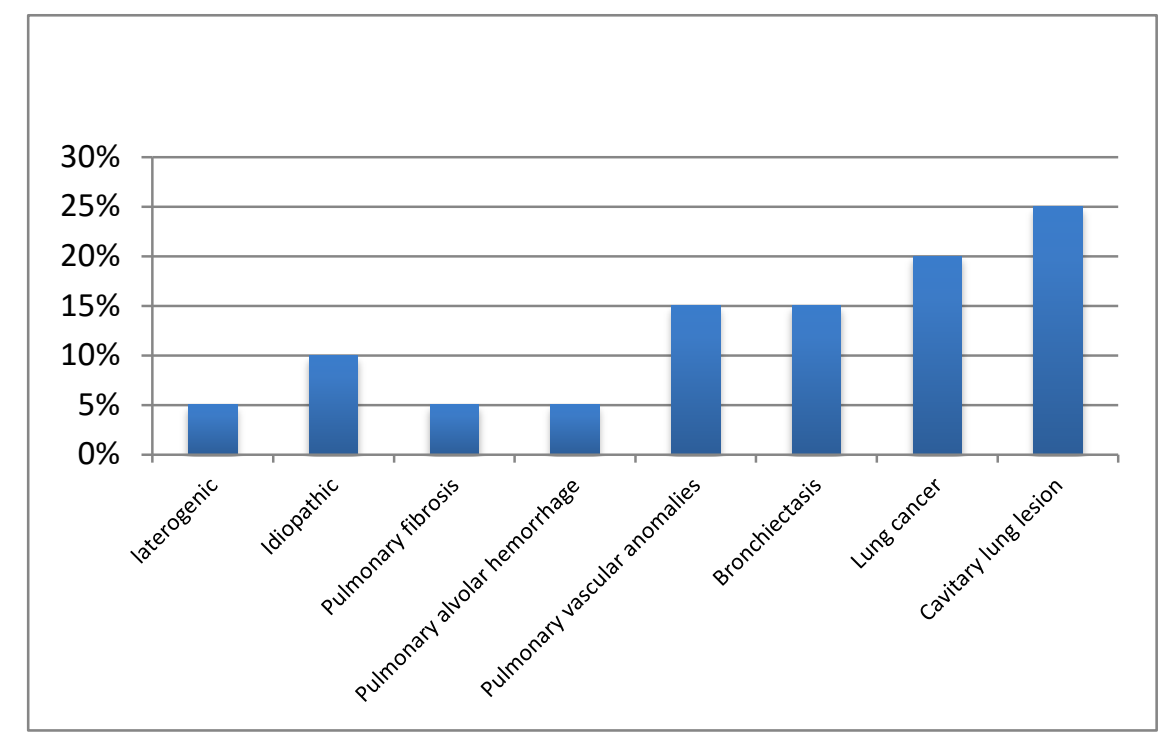

Diagram (2): Etiology of Hemoptysis in studied patients

Left lower lung lobe was the most commonly involved in our studied population whereas upper lobe pathologies were of $30 \%$. Focal pathologies were more frequent than its diffuse counterpart (Diagram 3).

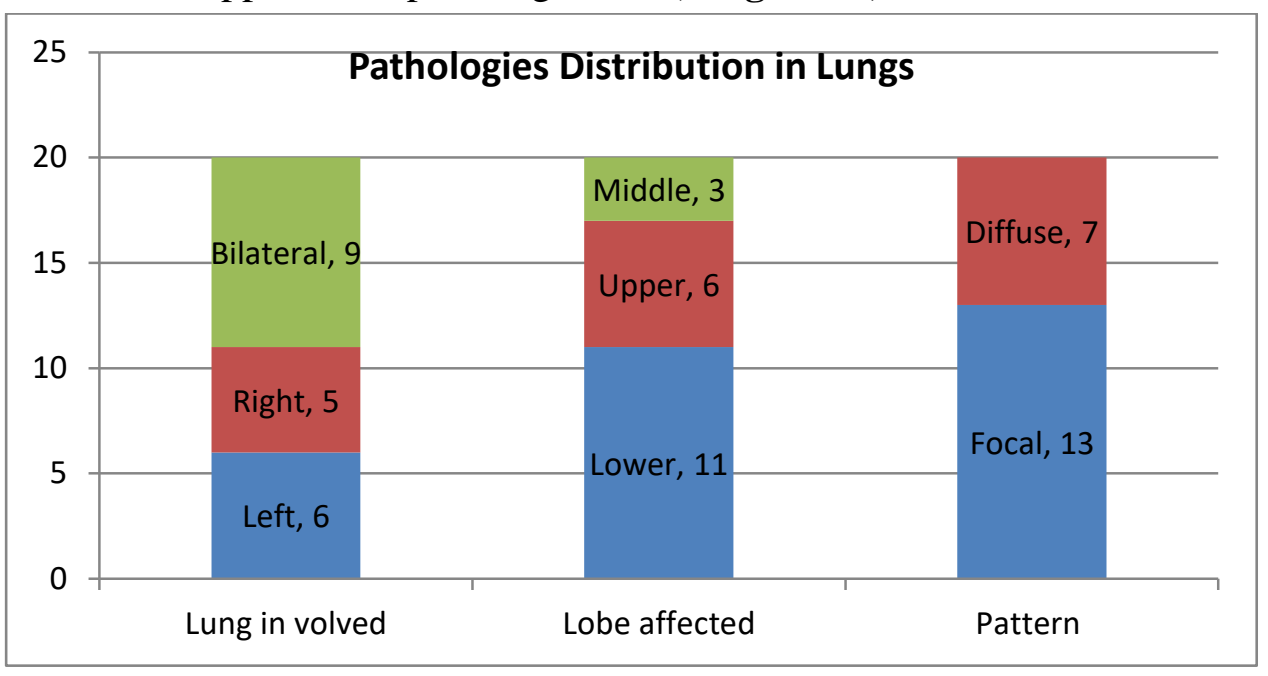

Diagram (3): Distribution of lung pathologies depicted in our study.

Right intercostobrachial trunk (IBT) and left bronchial artery were the most common bronchial configuration $(27.8 \%$ \& $25 \%)$ respectively. Three patients had extra- bronchial supply namely from internal mammary artery, Phrenic artery and subclavian artery (Table 2). 


\section{Laila Ahmad Abdurrahman et al.,}

Table (2): Number of the pathological arteries in catheter angiography in the studied patients.

\begin{tabular}{|c|c|c|}
\hline \multicolumn{2}{|c|}{ Pathological Arteries } & Number $(\%)$ \\
\hline \multicolumn{3}{|l|}{ Bronchial vessels } \\
\hline & Right BA & $7(19.4)$ \\
\hline & Left BA & $9(25 \%)$ \\
\hline & Right IBT & $10(27.77 \%)$ \\
\hline & Left IBT & $6(16.7 \%)$ \\
\hline & Common BA & $1(2.78 \%)$ \\
\hline \multicolumn{3}{|c|}{ Non bronchial vessels } \\
\hline & Internal mammary artery & $1(2.78 \%)$ \\
\hline & Phrenic artery & $1(2.78 \%)$ \\
\hline & Subclavian artery and its branches & $1(2.78 \%)$ \\
\hline
\end{tabular}

On conventional catheter angiography, pathological findings (90\%), followed by bronchial arterial hypertrophy, dilatation and parenchymal hypervascularity and tortuosity were the most common catheter neovascularization (80\%) (Table 3). Table (3): Conventional catheter angiography findings.

\begin{tabular}{|l|c|c|}
\hline & Catheter findings & Number (\%) \\
\hline $\mathbf{1}$ & BA Vessel Hypertrophy, dilatation \& tortuosity & $18(90 \%)$ \\
\hline $\mathbf{2}$ & Parenchymal hypervascularity/staining \& Neo-vascularization & $16(80 \%)$ \\
\hline $\mathbf{3}$ & Bronchopulmonary artery shunt & $2(10 \%)$ \\
\hline $\mathbf{4}$ & Active bleeding/ contrast extravasation & $2(10 \%)$ \\
\hline $\mathbf{5}$ & BA aneurysmal dilatation & $1(5 \%)$ \\
\hline
\end{tabular}

The clinical outcomes after BAE which was performed in 20 patients are demonstrated in table 4. Immediate success (one-month post-BAE) was achieved in $100 \%$ of patients in our study, while long term success (1-6 months post BAE) was achieved in $(75 \%)$. The severity of hemoptysis was assessed pre $\&$ post-BAE, and comparison was done. One patient $(5 \%)$ experienced another attack of moderate hemoptysis following BAE, however it was relieved after another two embolization sessions with one month apart. Similarly, 4 patients (20\%) experienced rebleeding, yet of mild amount in episodic pattern necessitating re-embolization in only two patients.

Table (4): Clinical Outcome of BAE in studied patients.

\begin{tabular}{|c|c|c|}
\hline \multicolumn{2}{|c|}{ Clinical Success } & Number (\%) \\
\hline 1 & Immediate success (1 month post-procedurally) & $20(100 \%)$ \\
\hline 2 & Long term success (1-6 months) & $15(75 \%)$ \\
\hline 3 & Recurrence & $5(25 \%)$ \\
\hline 4 & Mild hemoptysis & $4(20 \%)$ \\
\hline 5 & Moderate hemoptysis & $1(5 \%)$ \\
\hline 6 & Necessity for another embolization session & $4(10 \%)$ \\
\hline 7 & Mortality & $0(0 \%)$ \\
\hline
\end{tabular}

The complications of the procedure are enumerated in table 5. Minor complications were recorded in $60 \%$ of patients; three had post-procedural fever treated with IM antibiotics for 5 days, another patient presented with localized hematoma managed by sufficient compression for 1 hour. Eight patients complained of persistent severe pain for which night admission was required while being administered potent analgesics and non-steroidal anti- inflammatory agents. Neither major complication nor further bronchoscopic interventions occurred. 
Table (5): Post-procedural complication

\begin{tabular}{|c|c|}
\hline Minor Complication & Number (\%) \\
\hline Chest pain & $3(40 \%)$ \\
\hline Post-embolization syndrome (fever, nausea,) & $1(15 \%)$ \\
\hline Puncture site hematoma & $1(5 \%)$ \\
\hline Major Complications & 0 \\
\hline Spinal cord ischemia & 0 \\
\hline Reflux of embolizing agent into thoracic aorta
\end{tabular}

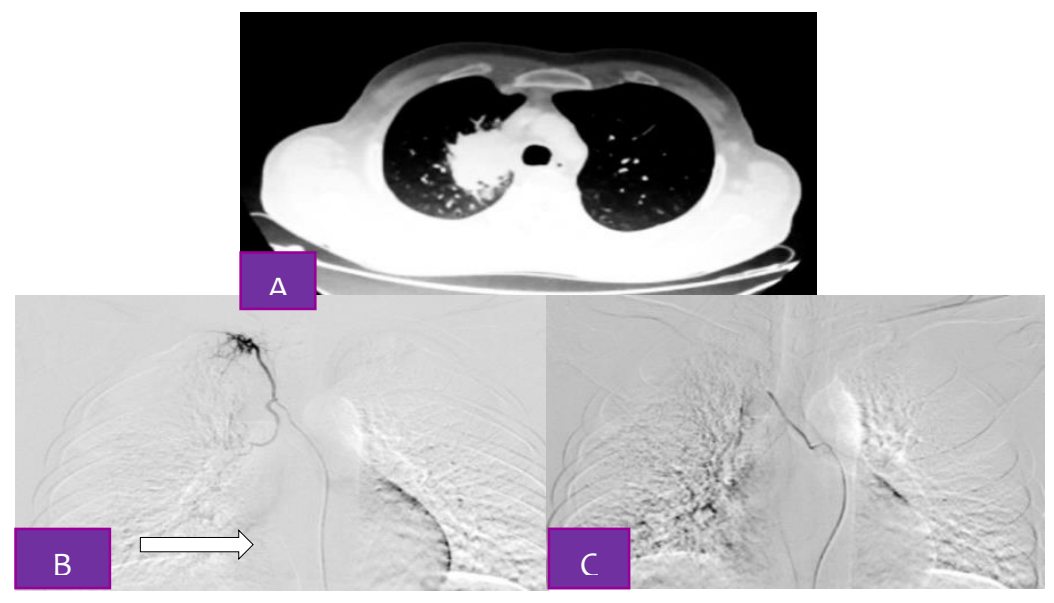

Fig. (1): (a) CT chest axial cuts at level of upper lobe revealed centrally located mass with speculated irregular margin at apical segment of right upper lobe suggesting a malignant behavior. (b) Bronchial Angiography exhibited a paralleling vascular tumoral blush fed by right intercostobrachial trunk (arrow). (c) Embolization was done via 500 $700 \mathrm{Mm}$ microspheres till disappearance of the blush and concomitant stasis at the feeding artery.

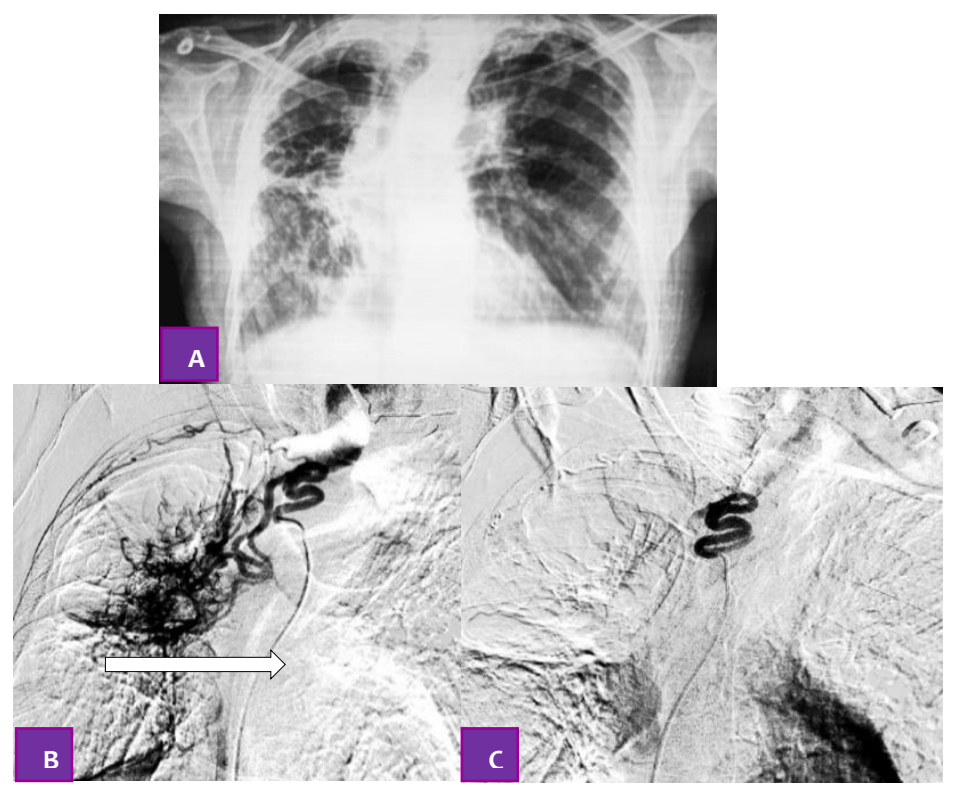

Fig. (2): (a) Chest X-ray revealed features of interstitial lung disease that is complicated with fibrotic band mainly on right middle lung segment exerting negative pressure effect on trachea and hilar structures (b) Digital subtraction angiography (DSA) revealed parenchymal neovascularization with bronchial vessels hypertrophy, tortuosity at right middle lung zone, fed by right intercostobrachial trunk (arrow) (c) Post-embolization angiogram revealed total disappearance of previously noted blush and stasis at the feeding artery following occlusion with microspheres particles of 300-500 M. 


\section{Laila Ahmad Abdurrahman et al.,}

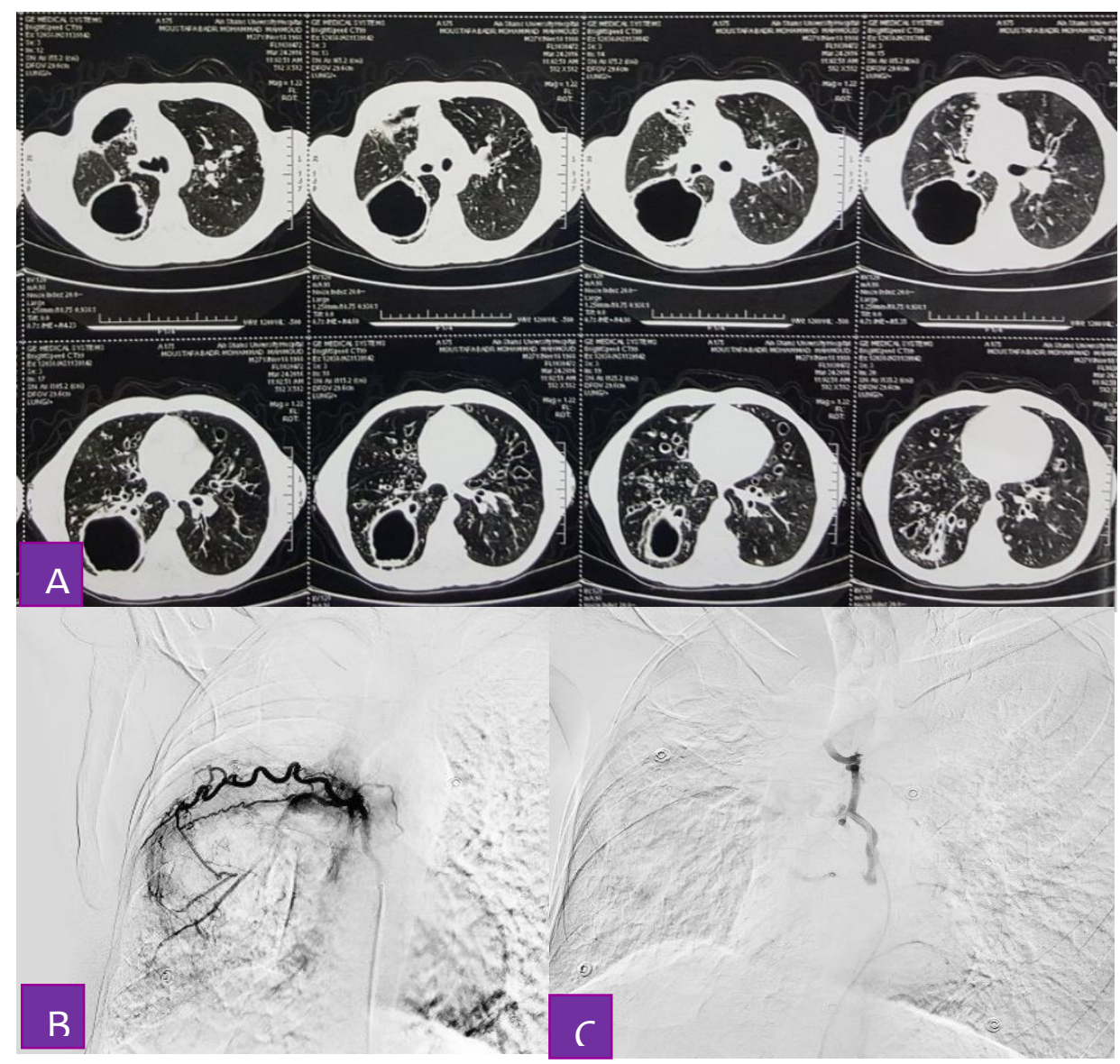

Fig. (3): (a) Admission CT revealed a relatively thick smooth walled cavitary lung lesion at the right lower lung lobe (b) bronchial angiography showed circumferential hypervascularity at lower lung zone consistent with the lung abscess (c) Post-embolization angiogram revealed total disappearance of previously noted blush and stasis at the feeding artery following occlusion with microspheres particles of 300-500 M. No other hemoptysis attacks were demonstrated through the course of follow-up.

\section{DISCUSSION:}

Life-threatening hemoptysis is one of the most serious emergencies in pulmonary medicine. Massive and untreated hemoptysis has a mortality rate of $>50 \%$. Lifethreatening hemoptysis definition in regard to amount of blood coughed varies in literature.

In our series it was defined as expectoration of $300-600 \mathrm{ml}$ of blood/ 24 hours or $200 \mathrm{ml}$ of blood/ hour with concomitant clinical deterioration manifested by hypotension (systolic BP less than $100 \mathrm{mmHg}$ ) and rapid drop in hemoglobin level of $1.0-2.0 \mathrm{mg} / \mathrm{dl}$ adding to failure of supportive or endobronchial measures to stop the bleeding.

Mal et al. ${ }^{(\boldsymbol{9})}$ described massive hemoptysis as expectoration of at least $200 \mathrm{ml}$ of blood/hour in a patient with normal lung function or production of at least $50 \mathrm{~mL}$ of blood/ hour in a patient with a chronic respiratory failure within a $24-\mathrm{h}$ period since decision to perform urgent $\mathrm{BAE}$ is not made always on basis of 24-h period.

In our sample population cavitary lung lesions (varying between lung abscess, aspergillosis and post-TB sequel) were the most common cause accounting for $25 \%$ of cases. Primary lung cancer and metastatic 
foci were represented by $20 \%$ of our patients. Seven patients presented to emergency department with ground glass opacity that was attributable to pulmonary vascular etiologies (3 patients) and bronchiectasis (3 patients) and pulmonary alveolar hemorrhage (one patient). Interstitial lung disease with subsequent pulmonary fibrosis was seen in one patient. Idiopathic and iatrogenic etiologies took place in 2 patients.

These results were nearly matched with Rashad et al. ${ }^{(10)}$ who attributed pulmonary TB to be the most common underlying cause of severe hemoptysis in their study. Agamy et $\boldsymbol{a l}^{\left({ }^{(5)}\right.}$ reported that pulmonary $\mathrm{TB}$, bronchiectasis, and mycetoma, accounted for $79 \%$ of all causes. Similarly; pulmonary tuberculosis accounted for $55 \%$ of patients at Shao et al. (11). On the contrary, lung malignancy was the most common cause of hemoptysis in $(46 \%)$ of patients according to Mohamed et al. ${ }^{(12)}$. In another study conducted by Pathak et al. ${ }^{(13)}$ cystic fibrosis was the most common etiology of hemoptysis in $(42 \%)$ of patients. This discrepancy might be related to varieties in the study population composition, and differences in geographic prevalence of tuberculosis and lung cancer. Therefore, further large-scale studies are warranted.

Samara et al. ${ }^{(14)}$ stated that the initial approach for massive hemptysis management is no different than for any other bleeding or hemodynamically unstable patient. Based on standard management protocols, physician's primary goals start by stabilizing the patient, securing the airway, identifying the bleeding site and efficiently containing the hemorrhage. Localization of the bleeding site is usually accomplished with imaging studies (chest x-ray, MDCT, CTA) and bronchoscopy.

Multi-detector CT angiography (MDCTA) usually portray a detailed road map of abnormal pulmonary vasculature, having a great impact on selecting proper management strategy in large number of patients as stated by Morita et al. ${ }^{(15)}$. The systemic bronchial arterial system is the source of bleeding in $95 \%$ of cases of hemoptysis; however depiction of pulmonary circulation as the origin of hemoptysis which is of utmost importance before sending patient to the angiounit as it will change dramatically the approach and management plan.

Chest CT scans have been reported to be superior to bronchoscopy in hemoptysis evaluation. Considered as the most sensitive diagnostic test; Hirschberg et al. ${ }^{(16)}$ stated that sole utilization of chest CT scan, yields positive result of $67 \%$, that increased up to 93\% upon combining CT scanning with bronchoscopy. A study by Lampmann and Tjan $^{(17)}$ of 50 patients undergoing embolotherapy with long-term follow-up found that bronchoscopy indicated the source of bleeding in 32 of 50 patients $(64 \%)$.

In a retrospective analysis on the management and prognosis of massive hemoptysis, Knott-Craig et al. ${ }^{(18)}$ found that rigid bronchoscopy was performed in 97 of 120 patients with hemoptysis localized the bleeding in only 42 (43\%). They also reported that the area of hemorrhage was able to be localized with some degree of confidence in only $63 \%$ of patients by a combination of radiologic evaluation, RBC labeled isotope scanning, and emergency endoscopy.

Visualization of the bronchial arteries was first documented in the late 1950s and early 1960s using nonselective aortography. Remy et al performed the first BAE in 1974 to control hemoptysis. In (1976), Wholey et al. ${ }^{(19)}$ published a series of 4 cases of successful BAE for the control of hemoptysis. Their embolization materials consisted of gelatin sponge strips (3 patients) and topical thrombin injection into the left bronchial artery (1 patient). 


\section{Laila Ahmad Abdurrahman et al.,}

This was followed by a large series by Remy et al in ${ }^{(20)}$ of 104 patients who were treated by embolization of both the bronchial and non-bronchial arteries to control hemoptysis. Forty-nine of these patients were managed during active hemoptysis, with immediate control of the hemorrhage seen in forty one patients $(84 \%)$. Subsequently, BAE was widely used, since nonoperable patients could be treated and other patients could be stabilized as a preoperative measure prior to definitive surgery $^{(2)}$.

\section{Regarding the source of bleeding:}

It is well-documented that bronchial arteries vary significantly in their numbers and sites of origin. Nearly $70 \%$ of bronchial arteries originate from the descending aorta between the levels of the fifth and sixth thoracic vertebrae. Cauldwell et al. in $(\mathbf{1 9 4 8})^{(21)}$ conducted a study upon 150 human cadavers and defined four types of bronchial anatomic variation where the most common type is single right bronchial artery with two left bronchial arteries (41\%). Up to $20 \%$ of bronchial arteries may have an aberrant origin (from other systemic arteries), and nearly $10 \%$ arise from the anterior surface of the aortic arch or the descending aorta.

A spinal artery can originate from a bronchial artery in up to $5 \%$ of patients, with right side being more common than the left side.

In our study a spinal artery was identified in nine of our patients, with five occurring on the left side and four occurring on the right side.

In our study, the origin of the bronchial arteries was observed in $(27.8 \%)$ of patients from the right intercosto-bronchial trunk, of direct origin from the left BA $(25 \%)$ and right BA (19\%), from the left IBT (17\%) whilst common trunk of both BAs was noted in( $3 \%$ ) which was in agreement with ElHusseiny et al. ${ }^{(22)}$. Non bronchial source of bleeding was noted in nearly $8 \%$ of patients distributed between internal mammary artery, phrenic and subcalvian arteries.

\section{Regarding the cause of hemoptesis:}

In our study bronchial vessels hypertrophy, dilatation and tortousity were the most common catheter angiographic findings and were demonstrated in $(90 \%)$ of our patients, which was quite similar to what reported by Anuradha et al. ${ }^{(23)}$ with $72 \%$ of their patients.

On the contrary, Agamy et al. ${ }^{(5)}$ reported that parenchymal hypervascularity, staining and neo-vascularization (defined as increased systemic arterial supply to an abnormal lung parenchyma with large caliber vessels) was the most common angiographic abnormality in $(98 \%)$ of patients.

Against our results which were $80 \%$. Kervancioğlu et al. ${ }^{(24)}$ reported bronchial arterial dilatation in $(53 \%)$ of their patients, and bronchial-to-pulmonary shunting in $(26 \%)$ of patients.

In our study, bronchopulmonary shunting, active bleeding represented the main angiographic abnormality in one-fifth of the patients.

Bronchial artery embolization is an efficacious technique in control of bleeding with immediate amelioration of clinical outcome as stated by Anuradha et al. ${ }^{(23)}$, Agamy et al. ${ }^{(5)}$, Rashad et al. ${ }^{(10)}$, Hwang et al. (25) and Shao et al. (11) who reported immediate success in $86 \%, 90 \%, 98 \%, 93 \%$ and $96 \%$ respectively.

In our study, all of our patients reported immediate relief of symptoms that persisted for 1 month post-procedurally.

\section{Regarding recurrence of bleeding:}

In the current study, despite satisfactory technical success rate denoted by disappearance of all angiographic 
abnormalities, still; recurrent hemoptysis remained a considerable problem. Recurrence of hemoptysis post-BAE was observed in 5 of 20 patients $(25 \%)$ who underwent BAE.

Which was higher than Agamy et al. ${ }^{(5)}$ who reported $10 \%$ recurrence necessitating re-embolization session in $8 \%$ of patients according to Agmy et al. series who repeated BAE in $(8 \%)$.

In this study, recurrent hemoptysis was moderate in one patient whilst was mild in 4 patients necessitating retreatment in 3 patients. Accordingly; our long-term hemoptysis control rate declined to $75 \%$ at the end of the follow-up 6 months period which was better than present in literature.

Anuradha et al. $^{(23)}$ reported that hemoptysis control rate declined progressively to $(51 \%)$ at the end of one year $\&$ to $(39 \%)$ at the end of two years, after which the condition stabilized. This difference in recurrence rates might be due to; the number of patients included in each study, the etiology of hemoptysis, the type of embolic material that had been utilized in embolization, either temporary or permanent, and the possible activity of the underlying etiology of hemoptysis adding to differences in the follow up period between these studies.

Hayakawa et al. ${ }^{(26)}$ have reported two peak times of bleeding recurrence. The first is from 1 to 2 months after the patient undergoes embolotherapy, which may reflect bleeding from non-bronchial systemic arteries that were not previously embolized. Others have noted the control of hemoptysis at 1 month in 51 to $85 \%$ of patients and control at 1 month in 52 to $82 \%$ of patients. The second peak for recurrence of hemoptysis is from 1 to 2 years after the patient undergoes embolotherapy. Recurrent bleeding after embolotherapy may indicate incomplete embolization of the bronchial arteries, the presence of non-bronchial systemic arteries, recanalization of embolized arteries, or collateralization due to continued pulmonary inflammation. In order to eliminate these types of recurrent hemorrhages, it is important to treat the underlying disease condition as well as to perform complete embolization of any systemic arteries that may be contributing to the blood supply.

In the series by Mal et al. (9) that included seven patients experienced recurrent hemoptysis within 1 month of undergoing BAE (16\%). Re-bleeding was noted very early in 4 patients; 10 days following embolotherapy for which second embolization session was successfully done with good functional outcome.

They further analyzed their outcomes by the type of embolization material that was used. In six patients who developed recurrent hemoptysis within 1 month post procedural, the embolization materials utilized included the following: gelatin sponge (2 patients); microspheres (2 patients); PVA (polyvinyl alcohol) (1 patient); and gelatin sponge plus bucrylate (1 patient). There was no further elaboration on this in the publication.

Swanson et al. (2) reported recurrent hemoptysis in 13 patients occurred in 13 patients from 54 patients. Patients were reembolized utilizing PVA in 2 patients and the rest with PVA and coils or gelatin sponge particles. One patient who was embolized with coils only, readmitted cause of recurrent hemoptysis and underwent successful re-embolization procedure.

On the other hand, Saluja et al. ${ }^{(27)}$ have suggested that coils should not be used for BAE as they cause proximal occlusion and do not allow for repeat embolization if necessary.

According to our study findings, we agree that coils will prevent repeat embolization of the same artery. However, they do provide a safe, thorough, and 


\section{Laila Ahmad Abdurrahman et al.,}

proximal occlusion of the artery, which can be difficult to achieve with injectable particles alone especially in difficult to access tortuous bronchial branches of whenever there is high flow bronchial fistula or dangerous anastmosis with spinal arteries. Moreover; It is still possible to embolize feeding collaterals if rebleeding develops. Polyvinyl chloride and coils provide permanent occlusion, while gelatin sponge particles are considered to provide temporary occlusion.

In our study; embospheres (300- 700 $\mu \mathrm{m})$ were utilized in 11 patients whilst PVAparticles were used in 7 patients. One case of pulmonary vascular malformation was managed by Gel foam to occlude the vascular bed followed by gel foam torpedoes to exclude the involve artery from the bronchial circulation. One case of pulmonary shunt was well-managed aided by deployment of several pushable coils. Readmitted patients $(n=5)$ were originally treated by Embospheres $(n=3)$ and PVA particles $(n=2)$.

\section{Regarding complication of BAE:}

Mortality attributable to bronchial artery Embolization has been scarcely depicted in literature.

In our study no deaths were noted.

Elhusseiny et al. ${ }^{(22)}$ reported one death during the course of his study in patient with right lobar mycetoma with recurrent massive hemoptysis for which BAE was successfully done, but death was attributed to rupture of dissecting abdominal aortic aneurysm.

The complication rate for BAE has diminished gradually over the years. During the early phases of selective bronchial arteriography, several patients developed transverse myelitis as a result of the use of nonionic contrast agents, more neurotoxin materials, and the inadvertent embolization of the spinal arteries. In Swanson et al. ${ }^{(2)}$ complications were predominantly catheterrelated varying between sub-intimal dissection, guidewire perforation, reflux of embolic agents into the aorta without adverse effects, transient dysphagia, pleuritic chest pain, shoulder pain, and groin hematoma.

In our study the technical related complications weren't evident owing to enormous advance in endovascular tools especially flow directed micro catheters and easily navigable microwires and more importantly embolization materials.

The most common complication in our study was chest pain represented by $40 \%$, followed by post-embolization syndrome comprising fever, nausea coupled by chest pain in $15 \%$ of patients. Post-procedural fever was controlled by intramuscular antibiotics. Persistent pain was wellcontrolled by potent analgesics and NSAIDS. Only one patient had self-limiting puncture site hematoma that was controlled by adequate compression with no necessity for surgical intervention.

Elhusseiny et al. ${ }^{(22)}$ reported femoral puncture site pain in $(56 \%)$ of patients, followed by chest pain (32\%), fever \& nausea (16\%). On the contrary, Agamy et $\boldsymbol{a l} .^{(2)}$ reported fever as the most common subacute complication post BAE in $(29 \%)$ of their patients, followed by chest pain $(5 \%)$ that were self-limiting with conservative management.

Iatrogenic bronchial dissection in 3 patients was also noted among the complications reported by Elhusseiny et $\boldsymbol{a l} .^{(22)}$ fortunately with no grave squeal. Moreover, hemoptysis in those patients stopped with no necessity for further intervention or bronchoscopic rescue maneuvers.

Various neurologic complications were stated by Elhusseiny et al. ${ }^{(22)}$ encompassing transient low back pain which was relieved by analgesics and right lower limb numbness and tingling attributable to femoral neuralgia during arterial puncture that persisted for 
about one week, yet were well-controlled thereafter via analgesics and vitamin B6 \& B12 injections.

During our study course no permanent major complications were noticed which might be attributable to super-selective catheterization of the bronchial arteries especially those harboring connections with spinal arteries.

\section{Conclusion:}

Advancements in angiographic equipment and technique continue to improve success rates, and with careful technique, it can be performed safely and with minimal risk. Embolization distal to the spinal artery may significantly decrease the number of complications and may allow a more thorough embolization.

\section{REFERENCES:}

1. Yoon W., Kim J., Kim Y., Chung T and Kang H. (2002): Bronchial and nonbronchial Systemic Artery Embolization for Life threatening Hemoptysis: A Comprehensive Review. Radiographics; 22: 1395-1409.

2. Swanson K., Johnson M., Prakash U., McKusick M., et al. (2002): Bronchial Artery Embolization. Experience with 54 Patients. Chest; 121:789-795

3. Burke C and Mauro M (2004): Bronchial Artery Embolization. Seminars in Interventional Radiology; 21(1): 43-48.

4. Sidhu M., Wieseler K., Burdick T. and Shaw D. (2008): Bronchial Artery Embolization for Hemoptysis. Seminars in Interventional Radiology; 25 (3): 310- 318

5. Agamy G., Wafy S., Mohamed S., Gad Y., et al. (2013): Bronchial and non-bronchial Systemic Artery Embolization for the management of Hemoptysis: Experience with 348 Patients. Vascular Medicince; 1-8.
6. Antonelli M., Midulla F., Tancerdi G., Salvator F., Bonci E., et al. (2002): Bronchial Artery Embolization for the Management of the non-massive Hemoptysis in Cystic Fibrosis. Chest; 21(1): 43-48.

7. Sopko D and Smith T (2011): Bronchial Artery Embolization for Hemoptysis. Seminars in Interventional Radiology; 28(1): 48-62.

8. Park H., Kim Y., Kim H., Zo J., et al. (2007): Bronchial Artery and Systemic Artery Embolization in the Management of Primary Lung Cancer Patients with Hemoptysis. Cardiovasc Intervent Radiol; 30:638-643.

9. Mal H., Rullon I., Mellot F., Brugie`re O., et al. (1999): Immediate and Long-term Results of Bronchial Artery Embolization for Life-Threatening Hemoptysis. Chest; 115:996-1001.

10. Rashad A., Amin M., Abd El-Azim A., Zidan M. and Mahmoud A. (2013): Percutaneous transcatheter vascular embolization for life threatening hemoptysis, Egypt. J. Chest Dis. Tuberc; 62:755-759.

11. Shao H., Wu J., Wu Q., Sun X., et al. (2015): Bronchial artery embolization for hemoptysis: a retrospective observational study of 344 patients, Chin. Med. J.; 128 (1): $58-62$.

12. Mohamed M, McMullen S, Hess LM, et al. (2019): Treatment decisions for advanced non-squamous non-small cell lung cancer: patient and physician perspectives on maintenance therapy. The Patient-PatientCentered Outcomes Research; 12(2):22333.

13. Pathak V., Stavas JM., Ford HJ., Austin CA. and Aris RM. (2016): Long-term outcomes of the bronchial artery Embolization are diagnosis dependent. Lung India; 33:3-8.

14. Samara K., Tsetis D., Antoniou K., Protopapadakis C., Maltezakis G. and Siafakas N. (2011): Bronchial artery embolization for management of massive cryptogenic hemoptysis: a case series. Journal of Medical Case Reports; 5:58. 


\section{Laila Ahmad Abdurrahman et al.,}

15. Morita Y., Takase K., Ichikawa H., et al. (2010): Bronchial artery anatomy: preoperative 3D simulation with multidetector CT. Radiology; 255 (3): 934-943.

16. Hirschberg B., Biran I., Glazer M., et al. (1997): Hemoptysis: etiology, evaluation, and outcome in a tertiary referral hospital. Chest; 112:440-444.

17. Lampmann L. and Tjan T. (1994): Embolization therapy in hemoptysis. Eur $\mathbf{J}$ Radiol; 18:15-19

18. Knott-Craig CJ., Oostuizen JG., Rossouw G., Joubert JR., Barnard PM. (1993): Management and prognosis of massive hemoptysis Recent experience with 120 patients. The Journal of Thoracic and Cardiovascular Surgery; 105(3):394-7.

19. Wholey MH, Chamorro HA, Rao G, et al. (1976): Bronchial artery embolization for massive hemoptysis. JAMA; 236(22):25014.

20. Remy J, Voisin C, Dupuis C, et al. (1974): Traitement des hemoptysies par embolization de la circulation systemique. Ann Radiol; 17:5-16

21. Cauldwell E., Siekert R., Lininger R., et al. (1948): The bronchial arteries: an anatomic study of 150 human cadavers. Surg Gynecol Obstet; 86:395-412.
22. Elhusseiny M., Abd-Elhafez S, Amer T., Eid R. and Suliman L. (2017): Bronchial artery embolization in management of hemoptysis: Safety and Efficacy. Egyptian Journal of Chest Diseases and Tuberculosis; 66:723-728.

23. Anuradha C., Shyamkumar N., Vinu M., et al. (2012): Outcomes of bronchial artery embolization for life-threatening hemoptysis due to tuberculosis and posttuberculosis sequelae, Diagn. Intervent. Radiol; 18:96-101.

24. Kervancioğlu C, Akyürek Ö, Berkalp B, et al. (2003): Altered coronary flow properties in diffuse coronary artery ectasia. American Heart Journal; 145(1):66-72.

25. Hwang H., Lee H., Choi J., et al. (2013): Risk factors influencing rebleeding after bronchial artery embolization on the management of hemoptysis associated with pulmonary tuberculosis, Tuberc. Respir. Dis; 74:111-119.

26. Hayakawa K., Tanaka F., Torizuka T., Mitsumori M., Okuno Y., et al. (1992): Bronchial Artery Embolization for Hemoptysis: Immediate and Long-Term Results. Cardiovasc Intervent Radiol; 15:154-159.

27. Saluja S., Henderson K. and White R. (2000): Embolotherapy in the bronchial and pulmonary circulations. Radiol Clin North Am; 38:425-448. 


\section{دور الحقن الثرياني عن طريق القسطرة التداخلية في علاج سعال الام الغزير}

المقدمة: سعال الدم الغزير هو حاله طارئة ومهددة للحياة مع معدل عالي في الوفيات. يسهم المنظار الثُعبي

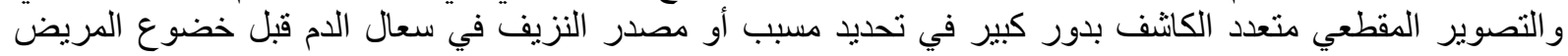
لتصوير الثريان الثعبي.

الهـف: التحقق من دور إصمام الثريان القصبي عبر القسطرة في إدارة نفت الدم الهائل.

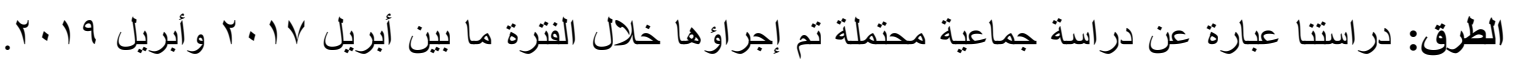

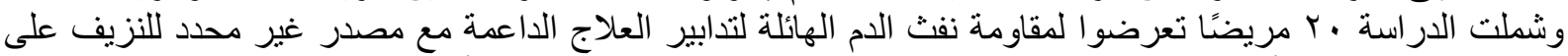

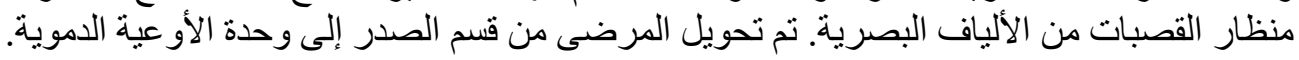

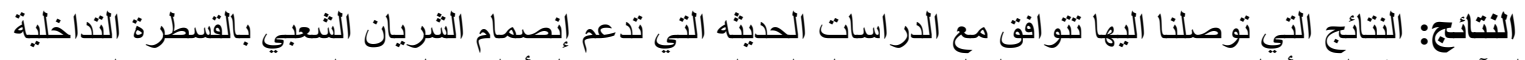

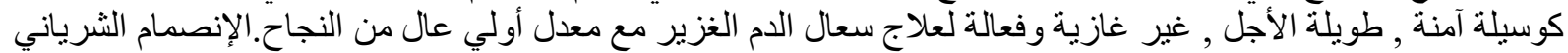

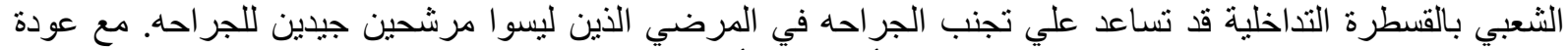

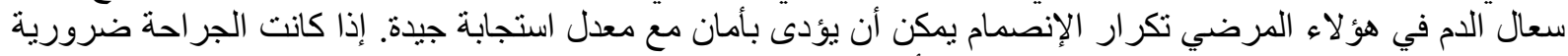

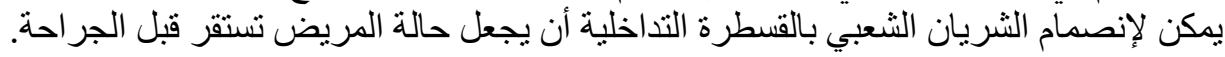

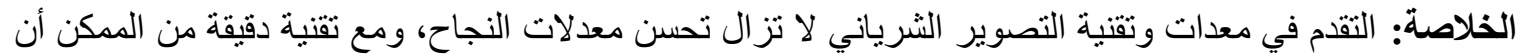

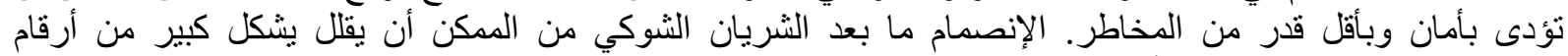
المضاعفات وقد يسمح بإنصمام أكثر شمو لأ لإن 